\section{Nonalcoholic fatty liver disease: another leap forward}

\section{Manal F. Abdelmalek}

In 2020, there have been substantial advances in nonalcoholic fatty liver disease mechanisms, diagnostics and treatment. Key developments include the identification of a cellular and tissue signature to provide new insights into pathophysiology, advancements in non-invasive diagnostics and publication of interim results of the first phase III trial to demonstrate improvement in hepatic fibrosis.

The field of nonalcoholic fatty liver disease (NAFLD) and its progressive form, nonalcoholic steatohepatitis (NASH), has, despite the challenges of the coronavirus disease 2019 (COVID-19) pandemic, continued to rapidly evolve in 2020. A change of nomenclature from NAFLD to metabolic associated fatty liver disease (MAFLD) has been proposed ${ }^{1}$, but the definition and terminology for clinical practice and clinical trials requires further deliberation and consensus. The vast number of publications and new therapeutic targets for NAFLD/NASH make it impossible to keep abreast of the relevant information. However, if we look at the studies published in late 2019 and in 2020, common themes emerge: a deeper understanding of disease pathogenesis, new and improved diagnostic and staging tools, and moving a step closer to FDA-approved therapies. Three papers in particular advanced these themes.

NAFLD ranges from simple steatosis to $\mathrm{NASH}$ with or without hepatic fibrosis in the absence of excessive alcohol intake. Whereas steatosis is not associated with an increase in liver-related morbidity or mortality, NASH can progress to more-severe stages such as cirrhosis and hepatocellular carcinoma, leading eventually to liver failure and liver transplantation ${ }^{2}$. Histopathological analysis remains the 'gold standard' to diagnose liver injury. However, invasiveness, risk and cost are issues. In addition, the poor inter-observer variability and modest intra-observer variability of liver biopsy analysis results in suboptimal reliability in measuring a treatment effect in clinical trials ${ }^{3}$.

High-definition medicine is emerging as an integrated approach to profile and restore an individual's health using multi-parametric analytical and therapeutic technologies. The $3 \mathrm{D}$ bile canalicular structure of the liver makes it an ideal organ for geometrical modelling and analysis of the liver microstructure and prediction of liver pathobiology.

To quantitatively characterize the transition from simple steatosis to early NASH, Segovia-Miranda et al. ${ }^{4}$ imaged and digitally reconstructed human liver tissue in both $2 \mathrm{D}$ and 3D from biopsy samples of healthy individuals as controls $(n=6)$, healthy individuals with obesity $(n=4)$, people with simple steatosis $(n=8)$ and those with early NASH $(n=7)$. Tissue sections were antigen-retrieved, stained for bile canaliculi, sinusoids, nuclei, lipid droplets and cell borders, and cleared and imaged at high resolution using multiphoton microscopy. Alterations in hepatocyte and lipid droplet size and distribution (along the central-to-portal vein axis) had the potential to discriminate simple steatosis from early NASH. Using a machine-learning framework, analysis of the bile canalicular features coupled with biliary flow dynamic simulations, which predicted increased pericellular biliary pressure and micro-cholestasis, consistent with elevated cholestatic biomarkers in patient sera, identified pathobiological processes in early NASH.

Such approaches could enable the development of a quantitative tool for early disease diagnosis, assess disease progression and/or tailor therapeutic options to the functional status of the liver tissue in a personalized approach. Indeed, evidence suggests that bile acid signalling and metabolism and bile acid homeostasis are disrupted in patients with NAFLD, and that drugs targeting the farnesoid X receptor (FXR)-fibroblast growth factor 19 axis or bile acid conjugates might be beneficial in the treatment of $\mathrm{NASH}^{5-7}$. This approach might help to identify new biomarkers for diagnosis (such as of simple steatosis, $\mathrm{NASH}$ and NASH with advanced hepatic fibrosis) and predict the functional status of the tissue, enabling identification of patients who will develop a negative clinical outcome from NAFLD/NASH.

A major priority and clinical necessity is the ability to identify patients with NASH who are at greater risk of progression to cirrhosis and who are candidates for clinical trials and emerging pharmacotherapies. Also in 2020, Newsome et al. ${ }^{8}$ published the FibroScan-AST (FAST) score, a score for the non-invasive identification of patients with significant NASH (NAFLD activity score $\geq 4)$ and liver fibrosis ( $\geq F 2)$ to identify those patients at increased risk of disease progression. This prospective study was conducted in a derivation cohort $(n=350)$ and validated in multiple global cohorts $(n=1,026)$ from North America, Europe and Asia. Five predictors of NAFLD and associated liver injury were considered: liver stiffness measurement by vibration-controlled transient elastography, controlled attenuation parameter, aspartate aminotransferase (AST), alanine aminotransferase (ALT) and AST:ALT ratio. A model employing liver stiffness measurement by vibration-controlled transient elastography, controlled attenuation parameter and AST had the best predictive properties.

Cut-off was 0.35 for sensitivity of $\geq 0.90$ and 0.67 for specificity of $\geq 0.90$ in the derivation cohort, leading to a positive predictive value of 0.83 (84 of 101), a negative predictive value of 0.85 (93 of 110) and an area under the ROC curve of 0.90 ( $95 \%$ CI 0.76-0.85). The researchers demonstrated that, if the FAST score was used to screen patients into clinical trials, the screen failure rate would drop from $50 \%$ with increasing FAST cut-offs, thereby facilitating the more accurate identification and selection of candidates for drug trials and reduce the number of unnecessary

\section{Key advances}

- Spatially resolved 3D models of human liver tissue identify quantitative multiparametric cellular and tissue signatures to define disease progression and provide new insights into nonalcoholic fatty liver disease pathophysiology 4 .

- The FibroScan-AST (FAST) score noninvasively identifies patients at risk of progressive nonalcohoholic steatohepatitis (NASH) for clinical trials and potentially in clinical practice, reducing unnecessary liver biopsies in patients unlikely to have significant disease ${ }^{8}$.

- The positive results of the phase III REGENERATE month-18 interim analysis demonstrated a dose-dependent improvement in NASH-related hepatic fibrosis, an outcome that is reasonably likely to render a clinical benefit ${ }^{5}$. 


\section{YEAR IN REVIEW}

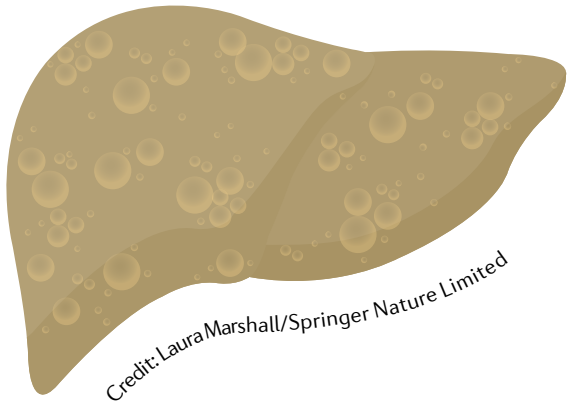

liver biopsies. When compared with Fib-4 and NAFLD fibrosis score, discrimination was significantly higher for the FAST score. Further research to evaluate the performance of the FAST score is now required to transition the use of such predictive models to primary care.

As the clinical burden of NASH has become increasingly clear, so too have challenges to developing effective treatment. There are currently no FDA-approved pharmaceutical therapies for NASH. However, 2020 marks a year of hope and cautious optimism as we begin to see change, despite multiple previous failures, in the therapeutic landscape of NASH. Late 2019 saw the publication of the first interim analysis of an ongoing multi-centre, randomized, placebo-controlled phase III trial evaluating obeticholic acid (OCA), a potent and selective FXR agonist, for the treatment of $\mathrm{NASH}^{5}$. Activation of the FXR can reduce hepatic fibrosis and inflammation ${ }^{5}$. FXR regulates lipid and glucose metabolism through a complex pathway involving multiple target genes, cytochromes and enzymes. But, ultimately, when activated, it converts cholesterol into bile acids, puts glucose into storage and reduces storage of fat. The REGENERATE study investigators recruited 931 patients with stage F2-F3 fibrosis (311 in placebo group, 312 in the OCA $10 \mathrm{mg}$ group and 308 in the OCA $25 \mathrm{mg}$ group). Fibrosis improvement was achieved in $12 \%, 18 \%$ and $23 \%$ of patients in the placebo, OCA $10 \mathrm{mg}$ and OCA $25 \mathrm{mg}$ groups, respectively ${ }^{5}$. Mild-to-moderate pruritus was observed in $19 \%, 28 \%$ and $51 \%$ of patients in the placebo, OCA $10 \mathrm{mg}$ and OCA $25 \mathrm{mg}$ group, respectively, with a similar incidence of serious adverse events across treatment groups. Patients receiving OCA were more likely to require a statin compared with placebo, which tempered the LDL increases. This study is ongoing to assess clinical outcomes.

Although the ultimate approval of OCA for NASH has been delayed by the COVID-19 pandemic and the FDA's request for additional post-interim safety and efficacy data, drug development pioneers of the NASH therapeutic landscape have unequivocally advanced the field. Stabilization and reversal of fibrosis, the primary predictor of liver-related and all-cause mortality, is paramount to improving clinical outcomes. Meeting this end point has proven to be a difficult task, with multiple agents failing to attain this elusive goal'; however, OCA, a first-in-class FXR agonist, is paving the way to finishing a race that started nearly 40 years ago, with the first reporting of NASH as a disease entity ${ }^{10}$.

These three studies each contribute to a complex field, whether it is with novel tools to facilitate clinical research and to better define disease mechanisms and efficacy end points or whether it is to bring us one step closer to new therapeutics. We must now utilize, further validate and build upon these findings and the lessons learned as we continue to aim to improve outcomes for patients with NAFLD/NASH.
Manal F. Abdelmalek

Division of Gastroenterology and Hepatology, Department of Medicine, Duke University, Durham,

NC, USA.

e-mail:manal.abdelmalek@duke.edu

https://doi.org/10.1038/s41575-020-00406-0

1. Eslam, M. et al. A new definition for metabolic dysfunction-associated fatty liver disease:

An international expert consensus statement. J. Hepatol. 73, 202-209 (2020)

2. Angulo, P. et al. Liver fibrosis, but no other histologic features, is associated with long-term outcomes of patients with nonalcoholic fatty liver disease. Gastroenterology 149, 389-397 (2015).

3. Davison, B. A. et al. Suboptimal reliability of liver biopsy evaluation has implications for randomized clinical trials. J. Hepatol. https://doi.org/10.1016/ j.jhep.2020.06.025 (2020).

4. Segovia-Miranda, F. et al. Three-dimensional spatially resolved geometrical and functional models of human liver tissue reveal new aspects of NAFLD progression. Nat. Med. 25, 1885-1893 (2019)

5. Younossi, Z. M. et al. Obeticholic acid for the treatment of non-alcoholic steatohepatitis: interim analysis from a multicentre, randomised, placebo-controlled phase 3 trial. Lancet 394, 2184-2196 (2019).

6. Harrison, S. A. et al. Efficacy and safety of aldafermin, an engineered FGF19 analog, in a randomized, double-blind, placebo-controlled trial of patients with nonalcoholic steatohepatitis. Gastroenterology https:// doi.org/10.1053/j.gastro.2020.08.004 (2020).

7. Iruarrizaga-Lejarreta, M. et al. Role of Aramchol in steatohepatitis and fibrosis in mice. Hepatol. Commun. 1, 911-927 (2017).

8. Newsome, P. N et al. FibroScan-AST (FAST) score for the non-invasive identification of patients with nonalcoholic steatohepatitis with significant activity and fibrosis: a prospective derivation and global validation study. Lancet Gastroenterol. Hepatol. 5, 362-373 (2020).

9. Rinella, M. E. \& Noureddin, M. STELLAR 3 and STELLAR 4: Lessons from the fall of Icarus. J. Hepatol. 73, 9-11 (2020).

10. Ludwig, J., Viggiano, T. R., McGill, D. B. \& Oh, B. J. Nonalcoholic steatohepatitis: Mayo Clinic experiences with a hitherto unnamed disease. Mayo. Clin. Proc. 55, 434-438 (1980)

\section{Competing interests}

Research funding (paid to institution): Allergan, Intercept, Madrigal, Viking Genfit Gilead, Galactin, Novo-Nordisk, Novartis, NGM Bio, Bristol-Myers Squibb, Genentech, Celgene, Poxel, Durect, Enyo, Inventiva, Enanta, Target Pharma, Boerhinger-Ingelheim, Hanmi. Advisory/consultancy: Allergan, Intercept, Madrigal, Novo-Nordisk, NGM Bio, Bristol-Myers Squibb, Inventiva, Boerhinger-Ingelheim, Promethera. Speaker's bureaus: Intercept, Clinical Care Options, Fishawack, Inc, Chronic Liver Disease Foundation, Medscape. 